\title{
An overview of current knowledge about cell-free RNA in amniotic fluid
}

\author{
Yong Wook Jung ${ }^{1}$, Yun Jeong Shin ${ }^{2}$, Sung Han Shim ${ }^{2,3}$, and Dong Hyun Cha ${ }^{1,2, *}$ \\ 'Department of Obstetrics and Gynecology, CHA Gangnam Medical Center, CHA University, Seoul, Korea \\ ${ }^{2}$ Genetics Laboratory, Fertility Center of CHA Gangnam Medical Center, CHA University, Seoul, Korea \\ ${ }^{3}$ Department of Biomedical Science, College of Life Science, CHA University, Seoul, Korea
}

Cell-free nucleic acids (cf-NAs) originate in trophoblasts and are detected in the maternal plasma. Using innovative bioinformatic technologies such as next-generation sequencing, cf-NAs in the maternal plasma have been rapidly applied in prenatal genetic screening for fetal aneuploidy. Amniotic fluid is a complex and dynamic fluid that provides growth factors and protection to the fetus. In 2001, the presence of cf-NA in amniotic fluid was reported. Amniotic fluid is in direct contact with the fetus and is derived from fetal urine and maternal and fetal plasma. Therefore, these genetic materials have been suggested to reflect fetal health and provide real-time genetic information regarding fetal development. Recently, several studies evaluated the global gene expression changes of amniotic fluid cell-free RNA according to gestational age. In addition, by analyzing the transcriptome in the amniotic fluid of fetal aneuploidy, potential key pathways and novel biomarkers for fetal chromosomal aneuploidy were identified. Here, we review the current knowledge of cell-free RNA in amniotic fluid and suggest future research directions.

Key words: Amniotic fluid, Transcriptome, Gene expression profiling, Prenatal diagnosis, Fetal development.

\section{Introduction}

In 1997, Lo et al. [1] reported the presence of fetal cell-free nucleic acid (cf-NA) in maternal plasma. Since this seminal discovery, there have been considerable achievements in the analysis and clinical applications of cf-NA in maternal blood. By adopting high-throughput sequencing technology, noninvasive prenatal screening of fetal aneuploidy using cellfree fetal DNA (cff-DNA) in maternal plasma has been rapidly integrated into antenatal care [2].

However, there are several limitations to the further application of cf-NA in maternal blood. First, cell-free fetal NA
(cff-NA) in the maternal plasma can become contaminated with maternal cf-NA. Most cf-NA in the maternal plasma originates from the mother. For example, fetal DNA accounts for only around 5-10\% of total cf-DNA present in the maternal plasma [3]. Second, the amount of fetal NA in the maternal plasma is low [4]. Third, cff-NA in the maternal blood does not originate from the fetus, but from trophoblasts that comprise the placenta [5].

Amniotic fluid is a complex and dynamic solution that protects the fetus and provides nutrients and growth factors for fetal growth. It changes as pregnancy progresses and provides crucial information to allow assessment of fetal maturity and

\footnotetext{
Received: 9 November 2016, Revised: 7 December 2016, Accepted: 8 December 2016, Published: 31 December 2016

${ }^{*}$ Corresponding author: Dong Hyun Cha, M.D., Ph.D.

Department of Obstetrics and Gynecology, CHA Gangnam Medical Center, CHA University, 566 Nonhyeon-ro, Gangnam-gu, Seoul 06135, Korea.

Tel: +82-2-3468-3132, Fax: +82-2-3468-2616, E-mail: chadh001@chamc.co.kr

Conflict of interest: The authors declare that they do not have any conflicts of interest.

(c) This is an open-access article distributed under the terms of the Creative Commons Attribution Non-Commercial License (http://creativecommons.org/licenses/by-nc/4.0/) which permits unrestricted non-commercial use, distribution, and reproduction in any medium, provided the original work is properly cited.

(c) Copyright 2016 by the Korean Society of Medical Genetics 
disease, such as fetal karyotyping in mid-trimester, diagnosis of intra-amniotic infection, and fetal lung maturity testing [6]. In addition, amniotic fluid (AF) contains cff-NA $[4,7]$. The potential advantage of cff-NA in AF is likely to originate from the fetus than the maternal plasma and placenta [8]. Cff-NA is more abundant in the AF than in the maternal blood [4]. Finally, AF cff$N A$ is considered to be relatively less contaminated by maternal cf-NA [4].

Most studies on the development of human fetuses have been performed by evaluating tissues obtained from human abortus and animal models, which mimic human fetus development, because of the difficulties in examining living human fetuses. However, cff-RNA in AF is considered to represent the real-time fetal physiology as the pregnancy progresses. Recent studies of AF cff-RNA have revealed novel information regarding the development of aneuploidy fetus [9-11]. This information may provide alternative approaches for the treatment of fetal aneuploidy in utero. However, studies of cf-RNA in AF are still in their infancy. Here, we summarize the current knowledge related to cf-RNA in AF and suggest future research directions.

\section{Cell-free Fetal RNA Was Extracted from Amniotic Fluid Supernatant}

Cff-DNA was first extracted in 2001 [4]. Prenatal molecular karyotyping was successfully performed with cff-DNA obtained from AF by hybridizing cff-DNA to microarrays for comparative genomic hybridization analysis [12]. However, cff-RNA was extracted from AF several years later. In 2005, Larrabee et al. [7] successfully extracted cf-RNA from AFS. The researchers used frozen, archived AFS from 6 male fetuses and 6 female fetuses as a pooled male control sample and pooled female control sample, respectively [13]. Five AF samples were obtained from 4 pregnant women; two had fetal hydrops and the other two had a fetus with twin-twin transfusion syndrome (TTS). The volumes of AF used for RNA were 60-120 mL. Of these 7 samples, RNA was successfully hybridized to microarray for analysis in 5 samples including the pooled male control sample. The authors showed that a $Y$ chromosome-specific probe was detected in only male fetuses by microarray analysis. Therefore, the extracted mRNA was considered to have originated from the fetus. In addition, the authors found that the genes involved in fetal development such as ossification and surfactant significantly increased as the pregnancy progressed. Compared with gene expression in pooled control samples, expression of Aquaporin $1(A Q P 1)$, a water transporter, was increased in TTS.
In combination with these results, the authors demonstrated the presence of cff-mRNA in AF, which can be used for microarray analysis, and suggested that cff-RNA in AF reflects differences in gene expression according to the fetal sex, gestational age when AF was obtained, and disease status between cases and pooled male controls.

The cf-RNA in plasma is stable because it is present in a particle-associated form, preventing cf-RNA from being degraded by nuclease in the plasma. The stability of cell-free mRNA in AF was examined by filtering AF and measuring the concentration of cf-RNA in filtered AF [7]. The study demonstrated that cf-RNA in AF was present in a particleassociated form as in the plasma. Therefore, AF cf-RNA is stable in the AF.

RNA sequencing is an innovative technology for accurately analyzing global gene expression in detail. Zwemer et al. [14] conducted a comparative analysis of RNA sequencing and gene expression microarray using the $\mathrm{cf}$ transcriptome. In the analysis, the authors demonstrated that gene expression levels were strongly and positively correlated. Microarrays provide more consistent information regarding gene expression for AF cf-RNAs. The application of RNA sequencing technology to AF cf-RNAs was limited by the presence of degraded cf-RNAs in the AF. However, RNA sequencing techniques can provide additional information related to alternative splicing.

\section{Where Does the Cell-free RNA in Amniotic Fluid Come from?}

Initial studies of AF cf-RNAs showed that placenta-specific genes were not present in the AF [15]. Based on this observation, the researchers concluded that the placenta, which directly contacts the AF, did not contribute AF cf-RNA. However, subsequent studies using techniques with improved efficiency to extract cell-free RNA and to amplify RNA in AF demonstrated that genes specific to the placenta were present in the AF [16]. In our study analyzing the cf-transcriptome in second-trimester $A F, 254$ genes were specifically enriched in the placenta [17]. We performed functional analysis of the Kyoto Encyclopedia Genes and Genomes (KEGG ) pathway for these genes. The results revealed that focal adhesion and extracellular matrix (ECM)receptor interaction pathways were enriched. Kim et al. [18] conducted a deep RNA sequencing study of 3 components in the human placenta (amnion, chorion, and decidua tissue) to examine the differences in global gene expression. They showed that focal adhesion and ECM-receptor interaction pathways 
were commonly enriched in these three placental tissues. Based on the results of these two studies, AF cf-RNAs may be present during placental development.

In addition, we found that cf-RNAs in AF were mostly annotated to the brain, endocrine organs, and blood components. The AF composition at 16-18 gestational weeks is similar to that of fetal plasma, because fetal skin is not yet keratinized [6]. These results suggest that cf-RNAs in the AF represent the origin of AF.

AF consists of amniocytes and acellular fluid. Amniocytes originate from all three germ layers of the embryo. Cell-free RNAs in AF may be derived from healthy cells through active secretion processes using exosomes or virtosomes and from necrotic cells through apoptosis or necrosis. Although the origin of cell-free fetal RNA in AF is not fully understood, AF cell-free RNA is considered to contain RNAs originating from both the fetus itself and amniocytes.

We examined the differences in the gene expression signature of RNA from amniocytes and AFS and determined whether cf-RNA in AF more adequately represents in utero fetal development than RNA from amniocytes. We performed comparative transcriptome analysis of cell-free RNA from second-trimester AF and from cultured amniocytes harvested during the second trimester [19]. In AFS and amniocytes, 3,072 and 15,633 genes were detected, respectively. Of those, 353 genes were specifically expressed in the AFS and 12,914 in amniocytes. We found that the uniquely expressed genes in AFS were associated with the brain, spleen, blood, heart, saliva, and testes. In contrast, the genes uniquely expressed in amniocytes were specifically enriched in the placenta, lung, epithelium, uterus, skin, and liver. Functional analysis of differentially expressed genes revealed that AF cf-RNAs were highly expressed genes in AF and were involved in embryonic and cardiovascular development, while enriched genes from amniocytes were associated with the pathways regulating infectious disease, cell death and survival, and protein synthesis. These results demonstrate that RNA in AF is a more valuable source of RNA for reflecting the fetal status. AF RNA also provides realtime information related to fetal development. Differences in transcriptome expression between AFS and cultured amniocytes indirectly revealed that the origin of cf-RNA in AF was not amniocytes.

However, few studies have evaluated the composition and origination of cf-RNA. Most studies evaluated the origin of cfRNA in AF indirectly through transcriptome analysis. Further studies are needed to directly determine the origin of cf-RNAs.

\section{Cell-free Transcriptome in Amniotic Fluid of Euploid Fetus Reflects Fetal Development}

The AF cell-free transcriptome of a second trimester euploid fetus was analyzed by Hui et al. [16] in 2012. The authors used publicly available gene expression data produced previously by their research group. The transcriptome data was obtained from the AF of 6 male and 6 female fetuses. A total of 476 genes were consistently expressed in all second trimester AFS. Functional analysis of the AF transcriptome revealed skeletal and muscular system tissue and hematologic system development and function as most significantly over-presented physiologic systems. The authors showed that cff-RNA in AF contained RNAs specific to the tissues that did not contact AF directly, including the central nervous system. Mammalian target of rapamycin signaling was identified as a critical pathway in the second trimester.

Our group performed transcriptome analysis with cf-RNAs in second trimester AF from 5 male and 9 female fetuses [17]. We identified 1,069 well-annotated genes in 14 samples. These genes were annotated with terms related to blood components, brain tissue, kidney, and endocrine organs including adrenal gland, thyroid, pancreas, ovary, and testes. As previously discussed, the AF composition before fetal skin keratinization is similar to that of fetal plasma. This result for AF transcriptome annotation revealed the origin of AF. KEGG pathway analysis identified several pathways related to brain development such as Parkinson's disease, Alzheimer's disease, and Huntington's disease and placenta development such as ECM receptor interaction and focal adhesion, which were over-presented.

Hui et al. [20] prospectively collected 16 AF samples form 8 women undergoing Cesarean section at term and 8 women who underwent second trimester amniocentesis. The researchers compared the transcriptome expression between the two groups to identify the tissue expression patterns and biological pathways in term AF. In term AF, cf-RNAs were enriched in the salivary gland, trachea, and kidney. This reflected that the major source of AF at term was secretions from the gastrointestinal tract and fetal urine. There were enrichments of upregulated genes at term in pathways related to immune function, digestion, respiration, carbohydrate metabolism, and adipogenesis in the functional analysis. The authors hypothesized that the upregulation of these pathways was preparation for postnatal energy requirements and thermal regulation. Inflammation and prostaglandin synthesis were activated, which were associated with labor induction. 
Combining these results, the researchers concluded that the cftranscriptome in AF reflects real-time gene expression according to fetal development.

Kamath-Rayne et al. [21] examined the AF transcriptome at different time points in pregnancy as follows; 4 samples from 18-24 gestational weeks, 6 from 34-36 gestational weeks, and 6 from 39-40 gestational weeks. For the analysis, the authors used an RNA sequencing technique to analyze transcriptome data. Expression of certain genes increased with advancing gestational ages, and these genes were enriched in several pathways associated with lipid and surfactant homeostasis, cell immune response, and response to growth factors. Gene expression which decreased with increased gestational ages was functionally associated with cell cycle and cell proliferation pathways, embryogenesis, and embryo development pathways. The authors identified tissue- or cell-specific markers in AF according to gestational ages of pregnancy. For example, $S C G B 3 A 1, D M B T 1$, and $A Q P 5$ were identified as specific markers of lung maturation.

Combining these results, AF cf-RNA reflects in utero fetal development and provides real-time genetic information according to fetal maturation.

\section{Transcriptome Analysis of Amniotic Fluid Cell-free Fetal RNAs for Fetal Aneuploidy}

\section{Down syndrome}

The first study examining how the expression of the cellfree fetal transcriptome in AF changes during pregnancy with aneuploid fetus was published in 2009 by Slonim et al. [9]. They examined the cf-transcriptome in AF obtained from 7 pregnant women with Down syndrome fetuses and 7 subjects with euploid controls matched for gender and gestational age. There was widespread differential expression of the cftranscriptome between Down syndrome and euploid fetuses. The authors performed functional analysis, which revealed that several functions in terms of oxidative stress, compensatory processes, and several developmental processes were disrupted in Down syndrome fetuses. Connectivity map analysis was used to confirm the results of functional analysis with differentially expressed genes.

Similar results were observed in previous studies in which brain tissues of Down syndrome fetus and those from animal models were used to examine the pathways involved in the development of Down syndrome [22-25]. Although previous studies identified functional pathways considered to be associated with Down syndrome development, the pathways were examined indirectly by using dead fetuses or animal models. However, studies of cff-RNA in AF may recapitulate the in utero development of living fetus. In addition, the study analyzing the cff-transcriptome identified candidate compounds such as celastrol, calmidazolium, verapamin, felodipine, and dimethyloxalyglycine to treat Down syndrome during fetal development by using connectivity map of differentially expressed genes. Calmidazolium is a calmodulin inhibitor. Verapamil and felodipine are calcium channel blockers. Dimethyloxalyglycine is a hydroxylase inhibitor. These compounds have been suggested as potential candidates for reversing the Down syndrome phenotype according to their analysis results.

\section{Edward syndrome}

Edward syndrome is a genetic disorder in which all or part of a third copy of chromosome 18 is present. Koide et al. [10] examined the cell-free transcriptome in 2nd trimester AF of five $47, X X+18$. Differentially expressed genes in the $A F$ transcriptome were compared with those in the AF of 6 euploid female controls. A total of 251 genes showed statistically significant differences in expression between the two groups. Of these genes, only 7 were located on chromosome 18. The study evaluating global gene expression in AF with Down syndrome showed similar results in that the expression levels of only 5 probe sets of 414 differed from those of the control [9]. Functional pathway analysis of 251 genes revealed that ion transport, DNA repair, T-cell mediated immunity, G proteinmediated signaling kinases, and glycosylation pathways were enriched. In addition, adrenal gland development was downregulated in trisomy 18 samples. Adrenal cortical hypofunction is a feature of trisomy 18 [26]. The authors suggested that the down-regulation of adrenal gland development pathways reflected the in vivo development of Edward syndrome.

Hui et al. [27] studied the cf-transcriptome in AF with Down syndrome and Edward syndrome. The authors re-evaluated the accumulated transcriptome data. Based on previously observed results showing that the cf-transcriptome in AF was highly enriched in the central nervous system, the authors focused on functional analysis with several bioinformatics resources. The analysis demonstrated that AF transcriptomes for the two different aneuploidies were enriched in the nervous system. To identify potential biomarkers for abnormal neurodevelopment, the authors narrowed differentially expressed genes into nervous system-specific genes showing different gene 
expression. In Down syndrome, 4 genes, SOX11, DAAM2, MEF2C, and CELSR2 were specifically expressed in the nervous system. In Edward syndrome, 6 genes were enriched in nervous system: PLEKHA4, PRPH2, GPM6Z, NEUROD2, SOBP, and PTPRD. There was no commonly dysregulated gene between the two aneuploidies.

\section{Turner syndrome}

Turner syndrome is a common sex chromosome aneuploidy with a partly or completely missing X-chromosome. There are several common clinical phenotypes including short stature, premature ovarian failure, webbed neck, and coarctation of the aorta. Massingham et al. [11] analyzed cf-RNAs obtained from five $45, X$ samples and compared the results with those from five $46, X X$ controls. In the analysis, 470 annotated genes were differentially expressed. A total of 272 genes increased and 198 genes decreased in Turner syndrome. Compared with the euploid controls, genes involved in the hematologic/ immunologic system were differentially expressed. The authors suggested that this dysregulation may be an early presentation of autoimmune dysregulation observed in later lifetime in women with Turner syndrome. NFATC3 gene expression was upregulated in Turner syndrome. NFATC3 was reported to be associated with perivascular tissue remodeling. Therefore, upregulation of NFATC3 may play a role in the development of coarctation of the aorta. In addition, the authors found that $\angle P L R$ and IGFBP5 dysregulation were associated with the phenotype of Turner syndrome, including hyperlipidemia and short stature, respectively.

\section{Fetal prematurity and twin-twin transfusion syndrome} Neonates born before 37 gestational weeks are considered preterm or premature. The risk of complication increases with preterm infants. The most common complications of preterm infants include respiratory support and gavage feeding because of delays in fetal lung and gastrointestinal system development. Kamath-Rayne et al. [21] analyzed the AF cf-RNAs of 6 late preterm infants. Two received respiratory support, two required gavage feeding, and one received both treatments. Although limited conclusions could be drawn because of the small sample size, the authors compared AF cf-RNA global gene expression of the preterm infants with co-morbidities with those of term infants. In the analysis, global gene expression in late preterm infants receiving gavage feeding showed fewer genetic markers for gastro-intestinal tract. Genetic markers for fetal lung were decreased in preterm infants who required respiratory support.
TITS is a complication resulting from an intrauterine blood transfusion from one of twin to the other in monozygotic twins with a monochorionic placenta. Preliminary studies of transcriptome analysis of cf-RNA were performed with pooled AF of TITS as previously described [13]. In the study, a water transporter, $A Q P 1$, was suggested to be a crucial gene in the development of polyhydramnios associated with TTS because $A Q P 1$ was increased by up to 18 -fold only in fetuses with TITS compared to that in the pooled control.

Long-term complications such as abnormal neurologic outcome and cardiovascular dysfunction are important in fetuses with TTS. In addition, the complexity of the disease and lack of an appropriate animal model to elucidate the pathogenesis of TTS lead researchers to examine the global gene expression in the AF of TTTS. In 2013, Hui et al. [28] examined the AF transcriptome in 8 recipient twins and 8 healthy singleton controls. There were 801 genes differently expressed between groups. The authors performed functional analysis of the differentially expressed genes. Functional analysis showed that the genes were enriched several physiologic systems including cardiovascular and nervous systems, which were associated with long-term complications of TTTS. The process of neurite outgrowth is impaired and Neurexin 3, which is essential for normal synapse development, was significantly decreased in TIS. In the cardiovascular system, NTR3, which results in cardiac anomalies in animal models was down regulated in TTS recipients.

\section{Maternal obesity}

Maternal obesity is of great concern as it adversely affects pregnancy outcomes, with effects such as preeclampsia, gestational diabetes, and large for gestational age infants. In addition, childhood obesity, metabolic syndrome, and adverse neurodevelopmental outcomes have been reported to be associated with maternal obesity during pregnancy. Edlow et al. [29] analyzed the global expression of AF cf-RNA in obese pregnant women to examine the underlying pathogenesis through which maternal obesity affects fetal development in utero. The authors recruited 8 obese (body mass index [BMI] $\geq 30$ $\left.\mathrm{kg} / \mathrm{m}^{2}\right)$ and 8 lean $\left(\mathrm{BMl}<25 \mathrm{~kg} / \mathrm{m}^{2}\right)$ women undergoing second trimester amniocentesis prospectively. The AF transcriptomes were compared between the two groups. A total of 205 genes were differentially expressed. Genes specific to the central nervous system were identified, including $A P O D, C A 11, P C L O$, $B C L 2, B C L 2 L 11, B C L 3$, and STK24 on apoptosis pathways were differentially expressed between two groups. Ingenuity 
pathway functional analysis revealed that cell death was most significantly enriched in molecular and cellular function. Activated upstream regulators of dysregulated genes between obese and lean women were estrogen receptor (ESR1/2), FBJ murine osteosarcoma viral oncogene homolog (FOS), and signal transducer and activator of transcription 3 (STAT3). These upstream regulators were involved in hormonal and inflammatory signaling pathways, leptin regulation, glucose homeostasis, and hepatic steatosis. This functional analysis suggested that the AF in obese pregnant women were in proestrogenic and pro-inflammatory milieu. Although there were several limitations such as the small sample size and lack of pregnancy outcomes and maternal medical conditions, the study indicated that maternal obesity affects fetal development as early as the second trimester. In addition, the authors suggested potential pathogenesis regarding how maternal obesity affects the fetus in utero.

\section{Conclusion}

Although it is difficult to obtain AF because of the invasiveness of amniocentesis, AF is an informative and valuable biologic sample. With the recent discovery of the presence of cf-RNA in $A F$, the importance of AF has become even more important. We summarized the current status of the field in evaluating the AF cf-RNAs in various AFsamples.

The origin of AF cf-RNA is not completely understood because few studies have provided direct evidence of the origin of cfRNA. However, current evidence indicates that the AF cf-RNAs originate from components producing the AF. Cf-RNAs reflect fetal development of the euploid fetus according to gestational ages. Transcriptome analysis of fetal aneuploidy demonstrated that a small number of regulators may be crucial for the pathogenesis of Down and Edward syndromes. In addition, potential compounds have been proposed by analyzing the global gene expression of cf-RNAs in AF. The AF transcriptome in subject with maternal obesity showed statistically significant gene expression changes. AF provides evidence for in utero fetal development in cases of maternal or fetal metabolic disease.

However, some challenges remain. First, the direct evidence regarding from where AF cf-RNAs originate and the proportion of AF cf-RNA that accounts for the fetal fraction should be determined. Second, most studies were conducted using bioinformatics analysis. Therefore, the provided data should be verified in in vivo or in vitro experiments. For examples, $A Q P 1$ has been suggested as a crucial gene for TIS pathogenesis based on analysis of the AF cf-transcriptome in TITS samples. Further studies of not only $A Q P 1$ but also several other biomarkers in vitro or in vivo are needed to confirm the importance and usefulness of these factors. Third, candidate disease for further application of cf-RNA technology should be developed. Numerous studies have proposed the clinical application of cfRNA in AF in various diseases. However, most studies did not provide direct evidence. It is not easy to obtain AF. Therefore, future studies should focus on diseases likely to be associated with cf-RNA. The authors suggest developing biomarkers or techniques to assess fetal lung maturity.

Much work is needed before the AF cf-transcriptome can be clinically applied. However, previous studies have shown promising results for the development of novel treatments or biomarkers for fetal disease.

\section{References}

1. Lo YM, Corbetta N, Chamberlain PF, Rai V, Sargent IL, Redman CW, et al. Presence of fetal DNA in maternal plasma and serum. Lancet 1997:350:485-7.

2. Mersy $E$, Smits $\sqcup$, van Winden LA, de Die-Smulders CE; SouthEast Netherlands NIPT Consortium, Paulussen AD, Macville MV, et al. Noninvasive detection of fetal trisomy 21: systematic review and report of quality and outcomes of diagnostic accuracy studies performed between 1997 and 2012. Hum Reprod Update 2013;19 318-29.

3. Lo YM, Tein MS, Lau TK, Haines CJ, Leung TN, Poon PM, et al. Quantitative analysis of fetal DNA in maternal plasma and serum: implications for noninvasive prenatal diagnosis. Am J Hum Genet 1998;62:768-75.

4. Bianchi DW, LeShane ES, Cowan JM. Large amounts of cell-free fetal DNA are present in amniotic fluid. Clin Chem 2001;47:1867-9.

5. Masuzaki H, Miura K, Yoshiura Kl, Yoshimura S, Niikawa N, Ishimaru T. Detection of cell free placental DNA in maternal plasma: direct evidence from three cases of confined placental mosaicism. J Med Genet 2004;41:289-92.

6. Underwood MA, Gilbert WM, Sherman MP. Amniotic fluid: not just fetal urine anymore. J Perinatol 2005;25:341-8.

7. Larrabee $\mathrm{PB}$, Johnson $\mathrm{KL}$, Peter I, Bianchi DW. Presence of filterable and nonfilterable cell-free mRNA in amniotic fluid. Clin Chem 2005; 51:1024-6.

8. Lun FM, Chiu RW, Leung TY, Leung TN, Lau TK, Lo YM. Epigenetic analysis of RASSF1A gene in cell-free DNA in amniotic fluid. Clin Chem 2007;53:796-8

9. Slonim DK, Koide K, Johnson KL, Tantravahi U, Cowan JM, Jarrah Z, 
et al. Functional genomic analysis of amniotic fluid cell-free mRNA suggests that oxidative stress is significant in Down syndrome fetuses. Proc Natl Acad Sci U S A 2009;106:9425-9.

10. Koide K, Slonim DK, Johnson KL, Tantravahi U, Cowan JM, Bianchi DW. Transcriptomic analysis of cell-free fetal RNA suggests a specific molecular phenotype in trisomy 18. Hum Genet 2011;129:295-305.

11. Massingham $\sqcup$, Johnson KL, Scholl TM, Slonim DK, Wick HC, Bianchi DW. Amniotic fluid RNA gene expression profiling provides insights into the phenotype of Turner syndrome. Hum Genet 2014;133:107582.

12. Larrabee PB, Johnson KL, Pestova E, Lucas M, Wilber K, LeShane ES, et al. Microarray analysis of cell-free fetal DNA in amniotic fluid: a prenatal molecular karyotype. Am J Hum Genet 2004;75:485-91.

13. Larrabee PB, Johnson KL, Lai C, Ordovas J, Cowan JM, Tantravahi U, et al. Global gene expression analysis of the living human fetus using cell-free messenger RNA in amniotic fluid. JAMA 2005;293:836-42.

14. Zwemer LM, Hui L, Wick HC, Bianchi DW. RNA-Seq and expression microarray highlight different aspects of the fetal amniotic fluid transcriptome. Prenat Diagn 2014;34:1006-14.

15. Bianchi DW. Gene expression analysis of amniotic fluid: new biomarkers and novel antenatal treatments. Clin Biochem 2011;44: 448-50.

16. Hui L, Slonim DK, Wick HC, Johnson KL, Bianchi DW. The amniotic fluid transcriptome: a source of novel information about human fetal development. Obstet Gynecol 2012;119:111-8.

17. Jung YW, Shim SS, Park JE, Sung SR, Shim SH, Park HR, et al. Analysis of the cell-free amniotic fluid transcriptome expressed during the euploid mid-trimester of pregnancy. Eur J Obstet Gynecol Reprod Biol 2016;203:94-8.

18. Kim J, Zhao K, Jiang P, Lu ZX, Wang J, Murray JC, et al. Transcriptome landscape of the human placenta. BMC Genomics 2012;13:115.

19. Kang JH, Park HJ, Jung YW, Shim SH, Sung SR, Park JE, et al. Comparative transcriptome analysis of cell-free fetal RNA from amniotic fluid and RNA from amniocytes in uncomplicated pregnancies. PLoS One 2015;10:e0132955.

20. Hui L, Wick HC, Edlow AG, Cowan JM, Bianchi DW. Global gene expression analysis of term amniotic fluid cell-free fetal RNA. Obstet Gynecol 2013;121:1248-54.

21. Kamath-Rayne BD, Du Y, Hughes M, Wagner EA, Muglia $\sqcup$, DeFranco $E A$, et al. Systems biology evaluation of cell-free amniotic fluid transcriptome of term and preterm infants to detect fetal maturity. BMC Med Genomics 2015;8:67.

22. Zana M, Janka Z, Kálmán J. Oxidative stress: a bridge between Down's syndrome and Alzheimer's disease. Neurobiol Aging 2007;28:648-76.

23. Lockstone HE, Harris LW, Swatton JE, Wayland MT, Holland AJ, Bahn S. Gene expression profiling in the adult Down syndrome brain. Genomics 2007;90:647-60.

24. Harashima C, Jacobowitz DM, Stoffel M, Chakrabarti L, Haydar TF, Siarey RJ, et al. Elevated expression of the G-protein-activated inwardly rectifying potassium channel 2 (GIRK2) in cerebellar unipolar brush cells of a Down syndrome mouse model. Cell Mol Neurobiol 2006;26:719-34.

25. Best TK, Siarey RJ, Galdzicki Z. Ts65Dn, a mouse model of Down syndrome, exhibits increased GABAB-induced potassium current. $J$ Neurophysiol 2007;97:892-900.

26. Turner M, O'Herlihy C. Adrenal hypofunction and trisomy 18. Obstet Gynecol 1984;63(3 Suppl):84S-5S.

27. Hui L, Slonim DK, Wick HC, Johnson KL, Koide K, Bianchi DW Novel neurodevelopmental information revealed in amniotic fluid supernatant transcripts from fetuses with trisomies 18 and 21. Hum Genet 2012;131:1751-9.

28. Hui L, Wick HC, Moise KJ Jr, Johnson A, Luks F, Haeri S, et al. Global gene expression analysis of amniotic fluid cell-free RNA from recipient twins with twin-twin transfusion syndrome. Prenat Diagn 2013;33:873-83.

29. Edlow AG, Vora NL, Hui L, Wick HC, Cowan JM, Bianchi DW. Maternal obesity affects fetal neurodevelopmental and metabolic gene expression: a pilot study. PLoS One 2014;9:e88661. 